\title{
Implementing Photodissociation in an Orbitrap Mass Spectrometer
}

\author{
Lisa A. Vasicek, ${ }^{1}$ Aaron R. Ledvina, ${ }^{2}$ Jared Shaw, ${ }^{1}$ Jens Griep-Raming, ${ }^{3}$ \\ Michael S. Westphall, ${ }^{2}$ Joshua J. Coon, ${ }^{2}$ Jennifer S. Brodbelt ${ }^{1}$ \\ ${ }^{1}$ Department of Chemistry and Biochemistry, The University of Texas at Austin, 1 University Station A5300, Austin, TX \\ 78712, USA \\ ${ }^{2}$ Departments of Chemistry and Biomolecular Chemistry, University of Wisconsin, Madison, WI 53706, USA \\ ${ }^{3}$ Thermo Fisher Scientific GmbH, Bremen, Germany
}

\begin{abstract}
We modified a dual pressure linear ion trap Orbitrap to permit infrared multiphoton dissociation (IRMPD) in the higher energy collisional dissociation (HCD) cell for high resolution analysis. A number of parameters, including the pressures of the C-trap and HCD cell, the radio frequency (rf) amplitude applied to the C-trap, and the HCD DC offset, were evaluated to optimize IRMPD efficiency and maintain a high signal-to-noise ratio. IRMPD was utilized for characterization of phosphopeptides, supercharged peptides, and N-terminal modified peptides, as well as for topdown protein analysis. The high resolution and high mass accuracy capabilities of the Orbitrap analyzer facilitated confident assignment of product ions arising from IRMPD.
\end{abstract}

Key words: Photodissociation, Orbitrap, Peptide, Protein, HCD cell

\section{Introduction}

$\mathrm{O}$ ver the past decade, both electron- and photon-based activation methods have emerged as versatile alternatives to collision-activated dissociation for fragmentation of peptides in proteomics applications [1-5]. Both infrared and ultraviolet photodissociation have proven to be successful tools for proteomic analysis using quadruple ion traps (QITs) [1], time-of-flight mass spectrometers [6, 7], and Fourier transform ion cyclotron resonance (FT ICR) instruments $[8,9]$; in the latter, the high resolving power and mass accuracy have been particularly valuable for assignment of sequence ions. Implementation of photodissociation on other high performance mass spectrometers is a compelling objec-

Lisa A. Vasicek and Aaron R. Ledvina, authors contributed equally to this work.

Electronic supplementary material The online version of this article (doi:10.1007/s13361-011-0119-7) contains supplementary material, which is available to authorized users.

Correspondence to: Joshua J. Coon; e-mail: jcoon@chem.wisc.edu, Jennifer S. Brodbelt; e-mail: jbrodbelt@mail.utexas.edu tive, especially with the growing adoption of Q-TOF [10] and Orbitrap [11, 12] type instruments for high throughput proteomics applications. Here we present the modification of an Orbitrap mass spectrometer to allow infrared multiphoton dissociation (IRMPD). We demonstrate a variety of applications that highlight the utility of IRMPD in combination with high resolution and mass accuracy Orbitrap mass analysis.

IRMPD was performed in the HCD cell of a modified hybrid mass spectrometer comprising both linear ion trap and Orbitrap mass analyzers (Figure 1). Typically utilized for beam-type $\mathrm{CAD}$, the HCD cell provides a convenient region for photoexcitation of ions that have been mass-selected in the forward linear ion trap and transferred to the HCD cell [13]. Briefly, the HCD manifold was modified via the addition of a ZnSe window coaxial with the HCD cell and opening an aperture $(\mathrm{d}=\sim 2.54 \mathrm{~mm})$ in its end plate electrode, allowing the irradiation of the HCD trapping region with IR photons (see Supplemental Figure 1). Additionally, a nitrogen gas line was installed in the C-trap (a region normally used for spatial compression and temporally compact transfer of the ion bundle into the Orbitrap), and the top and bottom of the C-trap were fitted with ceramic plates to decrease gas conductance out of 


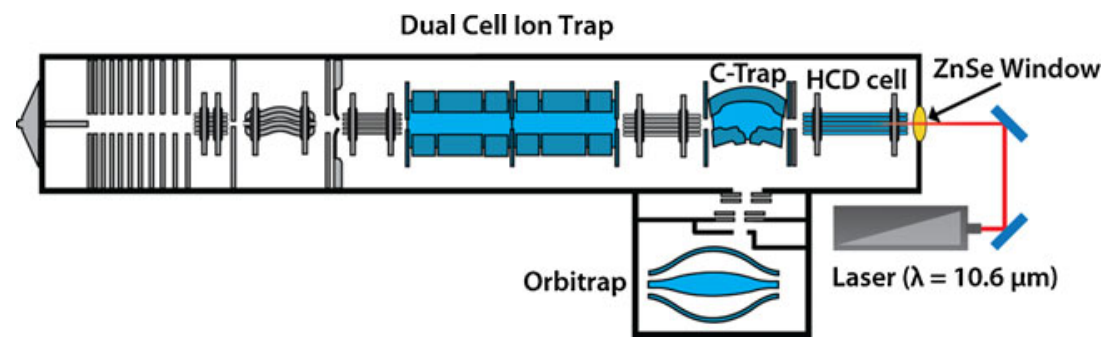

Figure 1. Schematic showing implementation of IRMPD in the HCD cell of a dual-cell linear ion trap-Orbitrap

the C-trap, reducing the gas flow needed to achieve desired trapping efficiencies in the C-trap while minimizing the $\mathrm{N}_{2}$ partial pressure in the QLT (quadrupole linear trap). Taken as a whole, these modifications allowed substantially independent control of nitrogen gas flow to both the C-trap and HCD cell. A $100 \mathrm{~W}$ Synrad $\mathrm{CO}_{2}$ laser was directed in the HCD cell using two optic mirrors mounted behind the HCD cell as shown in Supplemental Figure 1. The beam width of the laser is $2.2 \mathrm{~mm}$ with a beam divergence of $7 \mathrm{mR}$.

The optimization of nitrogen flow to the HCD cell and Ctrap is important for a number of reasons. IRMPD efficiency typically diminishes at higher pressures due to greater competition from collisional cooling $[14,15]$. The trapping efficiencies of both the C-trap and HCD cells are pressuredependent; with improved trapping efficiencies occurring at higher pressures, particularly for high $\mathrm{m} / \mathrm{z}$ ions. To evaluate the overall effects of gas flow (i.e., pressure) to both the HCD cell and C-trap on photodissociation efficiency, we evaluated three flow settings, one with reduced gas flow to both the C-trap and the HCD cell (P1), a second with a slightly increased flow to the C-trap while maintaining the reduced flow to the HCD cell (P2), and a third with no flow to the HCD cell and with the same increased flow to the C-trap as P2 (P3). Each flow setting led to corresponding changes in the pressures of the C-trap and HCD cell, and we observed notable changes in IRMPD signal levels and photodissociation efficiencies (Figure 2). The P1 setting led to the lowest absolute signal levels, nearly one order of magnitude lower than those observed using the P2 setting, but with the highest photodissociation efficiency (i.e., conversion of precursor ions to product ions). For the P2 setting, the photodissociation efficiency was relatively low due to collisional cooling, but the absolute signal level for the precursor ion was the highest. The P3 setting represented a compromise in the gas flow (and resulting pressures) that led to satisfactory precursor-to-product conversion efficiency and best product ion sensitivity, and therefore was used for all subsequent experiments.

In addition to pressure, the C-trap rf amplitude and HCD cell DC offset can influence overall IRMPD performance. As one parameter that defines the low-mass cut off, the C-trap rf amplitude is set as a function of the lower limit for the mass range selected through the user interface (also termed "first $\mathrm{m}$ / $z$ "). The C-trap rf amplitude also influences the efficiency with which product ions are trapped prior to injection into the Orbitrap for mass analysis. Optimization of this parameter was needed in response to the pressure changes in the C-trap and
HCD cell. As the C-trap rf amplitude varied from 600 to 2300 $\mathrm{V}_{p-p}$ (i.e., corresponding to a first $m / z$ range of 50-200 Th), the signal-to-noise $(\mathrm{S} / \mathrm{N})$ ratio increased substantially until leveling off at rf amplitudes above $1800 \mathrm{~V}_{p-p}$ (Supplemental Figure 2). $\mathrm{S} / \mathrm{N}$ was recorded for the most abundant fragment ion upon photoirradiation of a series of peptide precursor ions, and the averaged results are reported in Supplemental Figure 2. To allow trapping of as many of the low $\mathrm{m} / \mathrm{z}$ product ions as possible during IRMPD while still maintaining a high $\mathrm{S} / \mathrm{N}$ ratio, a C-trap rf amplitude of $1180 \mathrm{~V}_{p-p}$ was used for most of the remaining experiments. We varied the HCD cell rf amplitude from $100-500 \mathrm{~V}_{p-p}$, but observed no significant changes in photodissociation efficiency (data not shown), suggesting that over this range of rf amplitudes, the ion cloud maintains a diameter smaller than the laser beam diameter. Additionally, the HCD cell DC offset, the potential difference between the C-trap and HCD cell, plays a significant role in IRMPD performance as a result of the reduced pressures in the HCD cell. The HCD cell DC offset determines the kinetic energy with which ions are transferred from the C-trap to the HCD region. Under normal instrument operating conditions, this parameter modulates the collision energy during beamtype CAD, with greater HCD cell DC offsets providing greater collisional activation. At the reduced HCD cell pressures needed for efficient IRMPD, trapping of ions in the HCD cell is less effective. The HCD cell DC offset is normalized relative to precursor mass; as a result, no single HCD cell DC offset was optimal for the transfer of precursor ions. For the mass range of precursor ions utilized in this IRMPD study, HCD cell DC offsets of -8 to $-11 \mathrm{~V}$ relative to the QLT provided the best results in terms of the $\mathrm{S} / \mathrm{N}$ ratio of isolated precursor (results not shown). For HCD cell DC offsets greater than $-8 \mathrm{~V}$, the ions were not efficiently removed from the C-trap, and at offsets less than $-11 \mathrm{~V}$, ions underwent collisional activation upon injection to the HCD cell. At HCD cell DC offsets of -8 to $-11 \mathrm{~V}$, collisional activation prior to IRMPD was avoided while still efficiently extracting ions from the C-trap.

After optimization of the parameters summarized above, we utilized IRMPD in the hybrid Orbitrap system for a number of applications. Although conventional peptide cations exhibit relatively low IRMPD efficiencies due to significant competition with collisional cooling, phosphopeptides display ample IRMPD efficiencies even at mTorr pressures due to the high photoabsorptivities of $\mathrm{P}-\mathrm{O}$ bonds at $10.6 \mu \mathrm{m}[16,17]$. Phosphorylated peptides can be fully characterized and distinguished in a complex mixture with the phosphorylation 


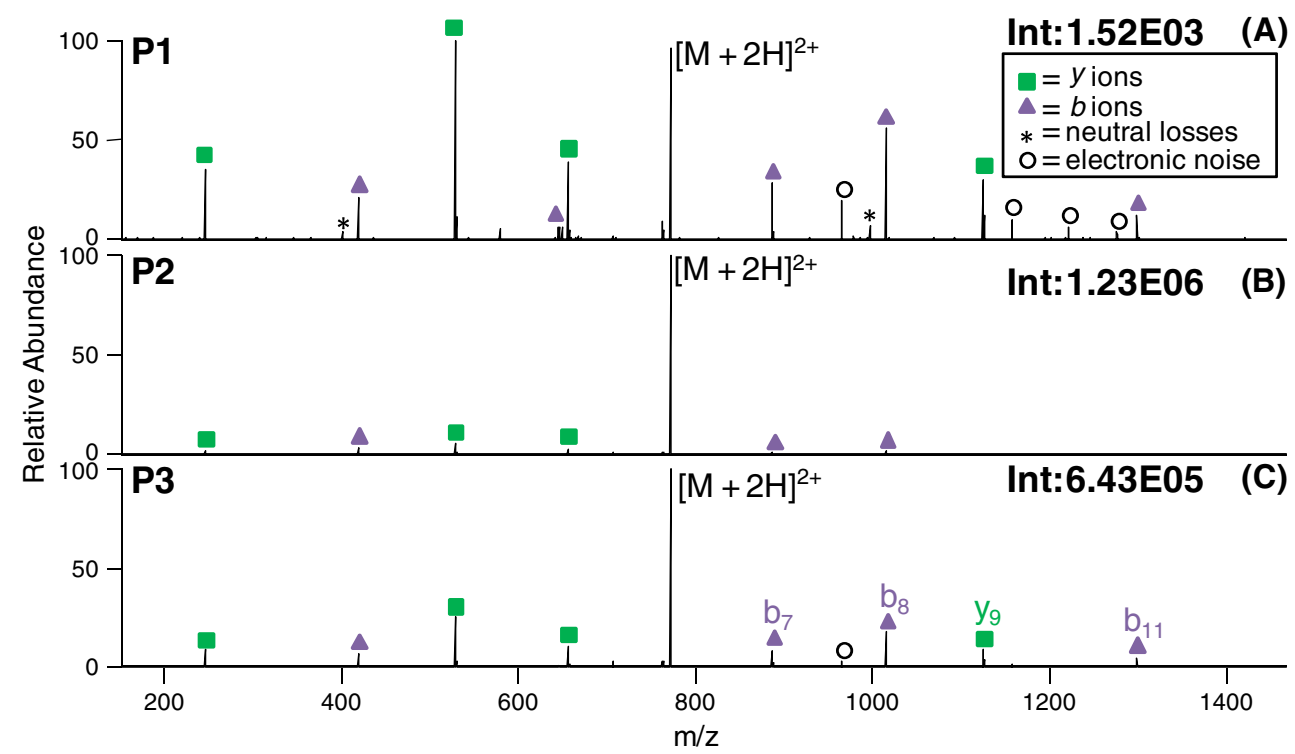

Figure 2. Effect of pressure on photodissociation efficiency and signal intensity. IRMPD of doubly charged phosphorylated peptide TSTEPQpYQPGENL, [M + 2H] $]^{2+},(10 \mu \mathrm{M}$ in 49.5/49.5/1 methanol/water/acetic acid), following $1 \mathrm{~ms}$ of $48 \mathrm{~W}$ irradiation at P1 (A), P2 (B), and P3 (C). rf Amplitude=1180 $\mathrm{V}_{p-p}$ (corresponding to first $\mathrm{m} / \mathrm{z}=100$ ), collision energy $=-11 \mathrm{~V}$

site being easily pinpointed through IRMPD following short irradiation times [18]. As shown in Figure 2c, the phosphorylated peptide, TSTEPpYQPGENL, yielded an array of diagnostic sequence ions after just $1 \mathrm{~ms}$ of irradiation. Production of $b_{7}, b_{8}, b_{11}$, and $y_{9}$ sequence ions, all of which retained the phosphoryl group, allowed facile localization of the site of phosphorylation. In addition to phosphorylated peptides, peptides specifically derivatized via attachment of an IR chromophore can likewise be dissociated with high efficiency. As we described previously, peptides can be derivatized at their Nterminus using a phosphono derivatization agent, PPITC, which adds a strong IR chromophore [19]. Upon IRMPD, PPITC derivatized peptides dissociated into $b$ and $y$ ions with preferential cleavage at the $y_{\mathrm{n}-1}$ bond, as previously reported, using as little as $5-10 \mathrm{~ms}$ of IR irradiation (see Supplemental Figure 3).

Ions in higher charge states undergo photodissociation more efficiently than those in lower charge states [20], an outcome largely attributed to the greater number of mobile protons in highly charged peptide ions. The abundances of ions in higher charge states can be enhanced via a "supercharging" method in which a small amount of a solvent additive, like $m$-nitrobenzyl alcohol, alters the charging dynamics of ESI droplets and the resulting ions $[21,22]$. This supercharging method also proved to be beneficial for improving the IRMPD efficiencies, as illus-

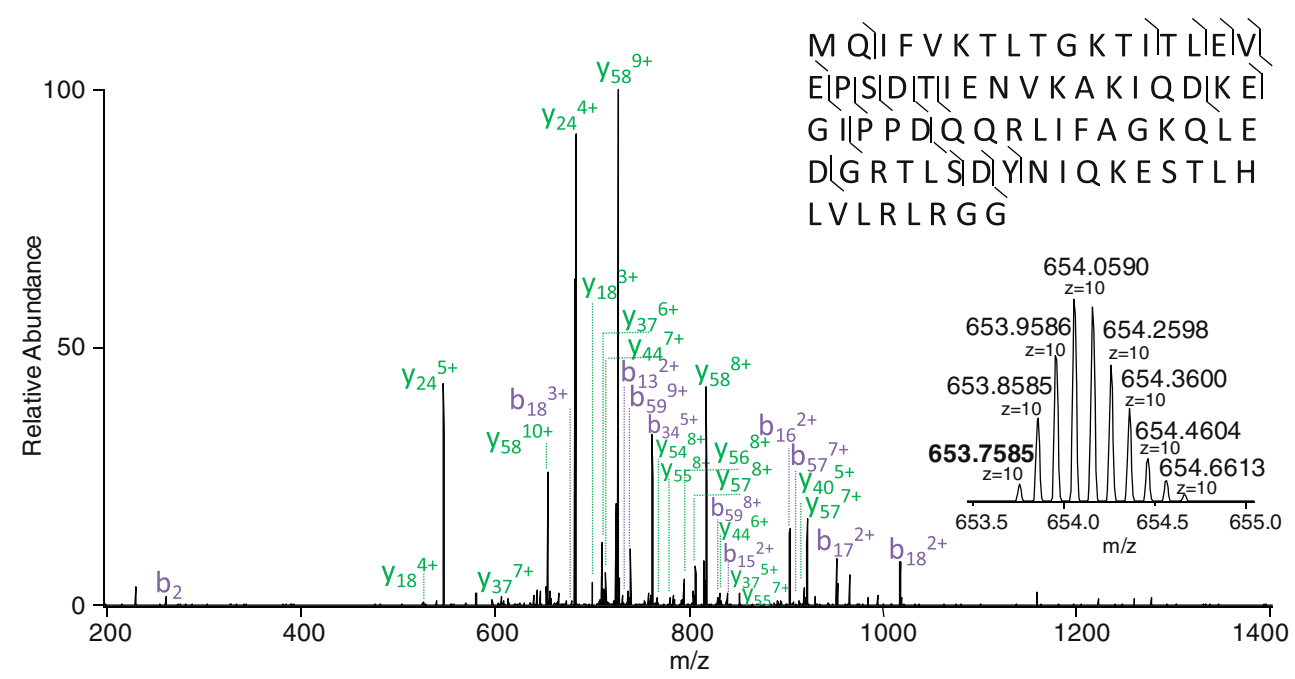

Figure 3. IRMPD of the $12+$ charged state of ubiquitin $(10 \mu \mathrm{M}$ in $49.5 / 49.5 / 1$ methanol/water/acetic acid) following $25 \mathrm{~ms}$ of $48 \mathrm{~W}$ irradiation, C-trap if amplitude $=2300 \mathrm{~V}_{p-p}$ (corresponding to first $\mathrm{m} / \mathrm{z}=200$ ), collision energy $=-11 \mathrm{~V}$. Inset shows baseline resolution of the $\mathrm{y}_{58}{ }^{10+}$ ion with a mass accuracy of $3.5 \mathrm{ppm}$ relative to the theoretical $\mathrm{m} / \mathrm{z}$ of 653.7562 
trated in Supplemental Figure 4 for supercharged peptide, ADSGEGDFLAEGGGVR, in the $3+$ charge state. A large array of $b$ and $y$ ions was generated after $8 \mathrm{~ms}$ of irradiation. The expected masses of the $b_{3}$ ion, 274.1042 Da, and $y_{2}$ ion, 274.1882 Da, (a difference of $306 \mathrm{ppm}$ from each other and $2.9 \mathrm{ppm}$ from the experimental values) make them indistinguishable in lower resolution ion traps. IRMPD in the HCD cell with subsequent mass analysis in the Orbitrap allowed unambiguous assignment of these ions. In some but not all cases, IRMPD results in a greater array of fragment ions than observed upon HCD, presumably due to consecutive IRMPD of primary fragment ions.

The enhanced resolution and mass accuracy is particularly beneficial for assignment of sequence ions upon IRMPD of whole proteins. Figure 3 shows the IRMPD spectrum of the $12+$ charge state of ubiquitin in which many of the fragment ions are highly charged, ranging from a singly-charged $b_{2}$ ion to the multi-charged $y_{70}{ }^{11^{+}}$ion. With the increased resolution afforded by Orbitrap mass analysis, all photodissociation product ions were confidently distinguished and assigned. For example, in the inset of Fig. 4, the $y_{58}{ }^{10+}$ ion was baseline-resolved with $3.5 \mathrm{ppm}$ mass accuracy. Interestingly, IRMPD also resulted in a greater degree of backbone cleavage selectivity compared to that obtained upon HCD with particular enhancement $\mathrm{N}$-terminal to proline and $\mathrm{C}$-terminal to glutamic acid residues. This phenomenon has been noted previously upon application of IRMPD in a dual pressure linear ion trap [23], albeit without high resolution or high mass accuracy ion assignments. A comparison of cleavage selectivity obtained upon IRMPD versus $\mathrm{HCD}$ for the 10+, 11+, and 12+ charge states of ubiquitin is shown in Supplemental Figure 5.

The results summarized here illustrate the practical rewards of photodissociation in an Orbitrap hybrid mass spectrometer, providing high resolution analysis on a variety of samples. Photodissociation was accomplished in the HCD cell in which a decrease in its gas flow (and pressure), along with a concomitant increase in the gas flow to the C-trap, most effectively balanced IRMPD efficiency and overall ion abundance (S/N). IRMPD does not outperform HCD, but it promotes an equally or more diverse array of fragment ions in some cases and demonstrates significantly enhanced performance for ions containing suitable chromophores. Maximizing both photodissociation and sensitivity requires further optimization of many of the parameters discussed.

\section{Acknowledgment}

Funding from the NSF (CHE-1012622, JSB), the Welch Foundation (F1155, JSB), NIH RO1 GM080148 (JJC) and PO1 GM081629 (JJC) is gratefully acknowledged.

\section{References}

1. Brodbelt, J.S., Wilson, J.J.: Infrared Multiphoton Dissociation in Quadrupole Ion Traps. Mass Spectrom. Rev. 28, 390-424 (2009)

2. Wiesner, J., Premsler, T., Sickmann, A.: Application of Electron Transfer Dissociation (ETD) for the Analysis of Post-Translational Modifications. Proteomics 8, 4466-4483 (2008)

3. Zubarev, R. Electron capture dissociation and other ion-electron fragmentation reactions. In Principles of Mass Spectrometry Applied to Biomolecules. Lifshitz, C.; Laskin, J., Ed. 2006, p. 475-517.

4. Ly, T., Julian, R.R.: Ultraviolet Photodissociation: Developments Towards Applications for Mass-Spectrometry-Based Proteomics. Angew. Chem. Int. Ed. 48, 7130-7137 (2009)

5. Coon, J.J.: Collisions or Electrons? Protein Sequence Analysis in the 21st Century. Anal. Chem. 81, 3208-3215 (2009)

6. Zhang, L., Reilly, J.P.: Peptide Photodissociation with $157 \mathrm{~nm}$ Light in a Commercial Tandem Time-of-Flight Mass Spectrometer. Anal. Chem. 81, 7829-7838 (2009)

7. Reilly, J.P.: Ultraviolet Photofragmentation of Biomolecular Ions. Mass Spectrom. Rev. 28, 425-447 (2009)

8. Mann, M., Kelleher, N.L.: Precision Proteomics: The Case for High Resolution and High Mass Accuracy. Proc. Natl. Acad. Sci. U.S A. 105, 18132-18138 (2008)

9. Eyler, J.R.: Infrared Multiple Photon Dissociation Spectroscopy of Ions in Penning Traps. Mass Spectrom. Rev. 28, 448-467 (2009)

10. Chernushevich, I.V., Loboda, A.V., Thomson, B.A.: An Introduction to Quadrupole-Time-of-Flight Mass Spectrometry. J. Mass Spectrom. 36, 849-865 (2001)

11. Hu, Q., Noll, R.J., Li, H., Makarov, A., Hardman, M., Cooks, R.G.: The Orbitrap: A New Mass Spectrometer. J. Mass Spectrom. 40, 430- 443 (2005)

12. Makarov, A., Denisov, E., Kholomeev, A., Balschun, W., Lange, O., Strupat, K., Horning, S.: Performance Evaluation of a Hybrid Linear Ion Trap/Orbitrap Mass Spectrometer. Anal. Chem. 78, 2113-2120 (2006)

13. Olsen, J.V., Macek, B., Lange, O., Makarov, A., Horning, S., Mann, M.: Higher-Energy C-Trap Dissociation for Peptide Modification Analysis. Nat. Methods 4, 709-712 (2007)

14. Black, D.M., Payne, A.H., Glish, G.L.: Determination of Cooling Rates in a Quadrupole Ion Trap. J. Am. Soc. Mass Spectrom. 17, 932-938 (2006)

15. Gardner, M.W., Smith, S.I., Ledvina, A.R., Madsen, J.A., Coon, J.J., Schwartz, J.C., Stafford, G.C., Brodbelt, J.S.: Infrared Multiphoton Dissociation of Peptide Cations in a Dual Pressure Linear Ion Trap Mass Spectrometer. Anal. Chem. 81, 8109-8118 (2009)

16. Flora, J.W., Muddiman, D.C.: Selective, Sensitive, and Rapid Phosphopeptide Identification in Enzymatic Digests Using ESI-FTICR-MS with Infrared Multiphoton Dissociation. Anal. Chem. 73, 3305-3311 (2001)

17. Flora, J.W., Muddiman, D.C.: Gas-Phase Ion Unimolecular Dissociation for Rapid Phosphopeptide Mapping by IRMPD in a Penning Ion Trap: An Energetically Favored Process. J. Am. Chem. Soc. 124, 6546-6547 (2002)

18. Crowe, M.C., Brodbelt, J.S.: Differentiation of Phosphorylated and Unphosphorylated Peptides by High-Performance Liquid Chromatography-Electrospray Ionization-Infrared Multiphoton Dissociation in a Quadrupole Ion Trap. Anal. Chem. 77, 5726-5734 (2005)

19. Vasicek, L.A., Wilson, J.J., Brodbelt, J.S.: Improved Infrared Multiphoton Dissociation of Peptides through N-Terminal Phosphonite Derivatization. J. Am. Soc. Mass Spectrom. 20, 377-384 (2009)

20. Madsen, J.A., Brodbelt, J.S.: Comparison of Infrared Multiphoton Dissociation and Collision-Induced Dissociation of Supercharged Peptides in Ion Traps. J. Am. Soc. Mass Spectrom. 20, 349-358 (2009)

21. Iavarone, A.T., Jurchen, J.C., Williams, E.R.: Supercharged Protein and Peptide Ions Formed by Electrospray Ionization. Anal. Chem. 73, 14551460 (2001)

22. Iavarone, A.T., Williams, E.R.: Supercharging in Electrospray Ionization: Effects on Signal and Charge. Int. J. Mass Spectrom. 219, 63-72 (2002)

23. Madsen, J.A., Gardner, M.W., Smith, S.I., Ledvina, A.R., Coon, J.J., Schwartz, J.C., Stafford, G.C., Brodbelt, J.S.: Top-Down Protein Fragmentation by Infrared Multiphoton Dissociation in a Dual Pressure Linear Ion Trap. Anal. Chem. 81, 8677-8686 (2009) 